\title{
Meta-analysis of Factor V Leiden G1691A polymorphism and osteonecrosis of femoral head susceptibility
}

\author{
XIFU SHANG, ZHENGLIANG LUO, XU LI, FEI HU, QICHUN ZHAO and WENZHI ZHANG \\ Department of Orthopedic Surgery, Anhui Provincial Hospital Affiliated to Anhui Medical University, \\ Hefei, Anhui 230001, P.R. China
}

Received January 29, 2013; Accepted March 25, 2013

DOI: $10.3892 /$ br.2013.93

\begin{abstract}
Testing for genetic risk associations between Factor V Leiden (FVL) and the osteonecrosis of femoral head $(\mathrm{ONFH})$ is common, however, inconsistent results have been previously obtained. To summarize results on the association of FVL mutation polymorphism with ONFH in various populations and to calculate the overall genetic risk factors, we performed a search of electronic databases including PubMed, Elsevier Science Direct, Chinese National Knowledge Infrastructure and the Chinese Biomedical Database to identify published studies correlating the FVL mutation with ONFH. Statistical analysis was performed using Review Manager (RevMan) version 5.0 and Stata statistical software (version 10). We identified 57 titles and included 7 studies comprising 481 cases and 867 controls in this meta-analysis. The groups were pooled, and a significant association between FVL mutation and increased ONFH was found $(\mathrm{OR}=4.55,95 \% \mathrm{CI}, 2.75-7.52, \mathrm{P}<0.00001)$. This meta-analysis demonstrated that FVL plays an important role in non-Asian populations. Large sample studies including different ethnic groups and age- and gender-matched groups, as well as multiple gene polymorphism detection should be considered to clarify the association of FVL mutation polymorphism and ONFH susceptibility in the future.
\end{abstract}

\section{Introduction}

Osteonecrosis of femoral head (ONFH) is a multifactorial degenerative bone disease, which may lead to the gradual destruction and collapse of the femoral head and secondary arthritis of the hip. Although idiopathic OFNH, steroid

Correspondence to: Mr. Zhengliang Luo, Department of Orthopedic Surgery, Anhui Provincial Hospital Affiliated to Anhui Medical University, 17 LuJiang Road, Hefei, Anhui 230001, P.R. China

E-mail: luoz10551@gmail.com

Key words: Factor V Leiden mutation, polymorphism, osteonecrosis of femoral head, meta-analysis treatment, alcohol consumption and trauma are considered the etiology of ONFH $(1,2)$, its pathogenesis remains to be clarified and several hypotheses have been proposed, including lipid dysfunction, increased apoptotic activity of osteocyte and osteoblast, disorder of bone homeostasis and intravascular and coagulation abnormalities (1,3). These multi-hits (the cause of the lipid dysfunction, increasing apoptotic activity of osteocyte and osteoblast, disorder of the bone homeostasis and the intravascular and coagulation abnormalities) result in compromised blood supply to the femoral head and this ischemic insult of the femoral head may result in ONFH. Therefore, the factors that induce a decrease in blood flow and vascular deficiency resulting in failure to obtain sufficient nutrients may be the final and of importance pathway of osteonecrosis.

Factor V (FV) is a pivotal auxiliary factor involved in the blood coagulation process. Following the proteolytic activation of $\mathrm{FV}$, the yield factor $\mathrm{Va}(\mathrm{FVa})$ forms the cofactor of the prothrombinase complex binding to the FXa, which regulates the formation of thrombin (4). Additionally, activated protein $\mathrm{C}$ (APC) modulates the generation of thrombin by inactivating the FVa. Factor V Leiden (FVL) gene mutation is a common polymorphism in $\mathrm{FV}$ that results in a guanine to adenine substitution at the nucleotide position 1691 (G1691A polymorphism), a missense mutation FV Arg506Gln (5). As a result, a single inactivation site of $\mathrm{FV}$ by activated protein $\mathrm{C}$ is altered, causing thrombophilia (an increased tendency to form thrombi) and leading to intravascular coagulation disorders. It was reported that FVL was associated with thromboembolic diseases such as symptomatic pulmonary embolism, deep vein thrombosis and myocardial infarction (6,7). Several case-control studies have been conducted to examine the association between FVL and ONFH (8-14). However, some studies have shown inconsistent or even contradictory results. Explanations for this discrepancy include small sample size, racial and ethnic differences, publication bias or uncorrected multiple hypothesis testing.

Meta-analysis is a statistical procedure for combining results from several studies to produce a single estimate of the major effect, thus enhancing the precision and statistical power (15). One of the advantages of meta-analysis is to increase sample size, which may control the probability of random error. Thus, meta-analysis is a useful and rigorous 
method to summarize different studies that have inconsistent results. With this as a background, we performed this meta-analysis to assess the association of FVL with the risk of ONFH in the published studies.

\section{Materials and methods}

Search strategies and criteria. A systematic literature search was conducted using the following electronic databases: PubMed, Elsevier Science Direct, Chinese National Knowledge Infrastructure (CNKI) and the Chinese Biomedical Database (CBM) prior to December, 2012, with the key words 'factor V' or 'Leiden' and 'osteonecrosis' as both medical subject heading (MeSH) terms and text words. No limitation on the language was applied.

The included criteria were summarized as follows: i) the study had already been published; ii) the study provided original data on the association of FVL and ONFH using a case-control study design; iii) enough data were provided to calculate the rate difference with a confidence interval (CI) and P-value and; iv) the genotype frequencies conformed to the Hardy-Weinberg equilibrium (HWE) among the controls. Exclusion criteria were: i) studies that contained overlapping data; ii) studies that were case reports and; iii) studies in which family members had been studied because their analysis is based on linkage consideration. An additional manual search of some relevant journals was conducted after perusal of the bibliographies of retrieved papers and review articles.

Data abstraction. Eligible publications were selected strictly according to the inclusion criteria listed above and all relevant data regarding first author's name, year of publication, patient demographic ethnicity, total number of cases and controls and genotyping information were independently extracted and recorded by two authors. Disagreements regarding extracted data were resolved by discussion with our research team. Different ethnicities were classified as non-Asian and Asian.

Meta-analysis methods. We examined the odds ratio (OR), i.e., the ratio of the odds of an event occurring in a case group to the odds of that event occurring in the control group and the respective $95 \%$ confidence intervals (CIs) by comparing FVL with the wild homozygotes GA + AA vs. GG. The within- and between-study variation or heterogeneity was also assessed using the $\chi^{2}$ test-bases Q-statistic and $\mathrm{I}^{2}$-statistic $(16,17)$. If a significant $\mathrm{Q}$-statistic $(\mathrm{P}<0.10)$ indicated heterogeneity across studies, the random-effects model was used for meta-analysis. Otherwise, the fixed-effects model was used. The random-effects model was used to take into account the possibility of heterogeneity between studies, while the fixed-effects model assumes all of the studies are estimating the same underlying effect and considers only within-study variation. The $\mathrm{I}^{2}$-statistic measures the degree of inconsistency in the studies by calculating what percentage of the total variation across studies is due to heterogeneity rather than by chance (18). Additionally, in the presence of $(\mathrm{P}<0.10$ or $\mathrm{I}^{2}>50 \%$ ) or not, the overall or pooled estimate of risk (OR) was obtained by using the DerSimonian-Laird random-effects model or using the Mantel-Haenszel fixed-effects model. The

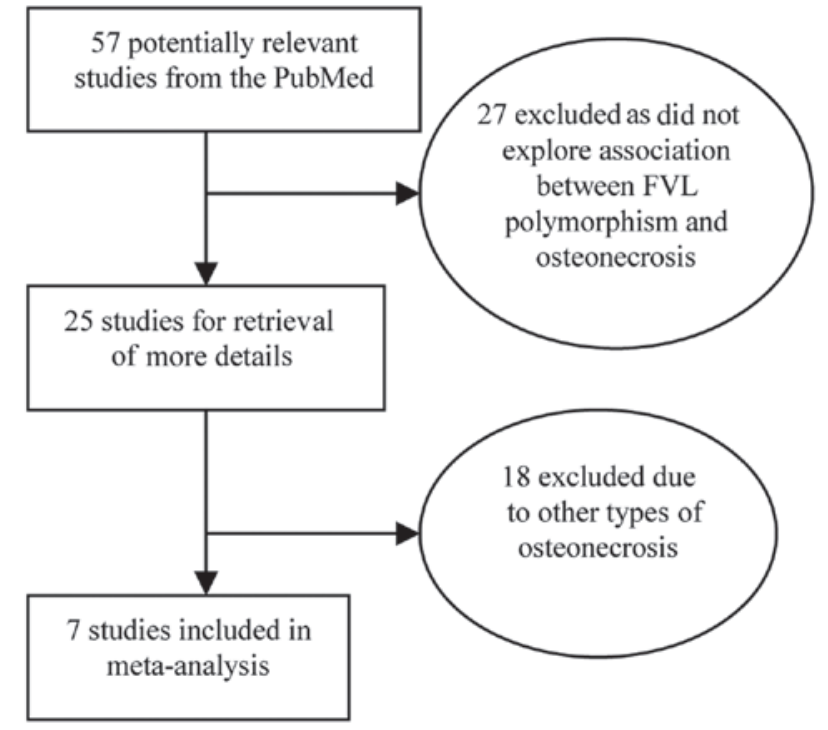

Figure 1. Flow chart of the studies selected using PubMed.

$\chi^{2}$ test was used for the HWE of genotypes in the control group of each study.

Publication bias was assessed by funnel plots, in which the standard error of $\log (\mathrm{OR})$ of each study was plotted against its $\log (\mathrm{OR})$. The funnel plot asymmetry, which suggests a possible publication bias, was examined by the method of Egger's linear regression test (15). The significance of the intercept was assessed by the Student's t-test, as previously mentioned (15). $\mathrm{P}<0.05$ was considered representative of statistically significant publication bias.

The meta-analysis was performed using the Review Manager software (ver.5.0, Copenhagen: The Nordic Cochrane Centre, The Cochrane Collaboration, 2008) and Stata statistical software (version 10) (Stata-Corp LP, College Station, TX, USA). All of the $\mathrm{P}$ values were two sided and $\mathrm{P}<0.05$ was considered to indicate a statistically significant difference.

\section{Results}

Characteristics of eligible studies. The characteristics of the 7 studies included in the present meta-analysis are provided in Table I (8-14). We reviewed 57 potentially relevant citations and abstracts obtained after a search of PubMed was conducted (Fig. 1). Of these, 27 studies were excluded as they did not survey the association between FVL polymorphism and osteonecrosis, thus 25 studies were screened for full publication review. Following a detailed evaluation of the remaining 25 studies, another 18 studies were deleted as they did not conform to the definition of ONFH (Fig. 1). The search performed in the Elsevier Science Direct, CNKI and CBM databases did not yield any additional studies not indexed by PubMed. A total of 7 studies were included in the current meta-analysis.

Evaluation of publication bias. Funnel plots and Egger's test were performed to access the publication bias of studies included in this meta-analysis. The shape of the funnel plot was not obviously asymmetrical in distribution for GA + AA vs. GG (Fig. 2). Furthermore, the Egger's regres- 
Table I. Characteristics of the studies included in the present meta-analysis.

\begin{tabular}{|c|c|c|c|c|c|c|c|c|c|}
\hline \multirow[b]{2}{*}{ First author } & \multirow[b]{2}{*}{ Year } & \multirow[b]{2}{*}{ Country } & \multicolumn{3}{|c|}{ Case } & \multicolumn{3}{|c|}{ Control } & \multirow[b]{2}{*}{ Refs } \\
\hline & & & Wild & Leiden & Total & Wild & Leiden & Total & \\
\hline Bjorkman & 2004 & Sweden & 54 & 9 & 63 & 271 & 11 & 282 & 8 \\
\hline Celik & 2006 & Turkey & 10 & 1 & 11 & 35 & 4 & 39 & 9 \\
\hline Ekmekci & 2006 & Turkey & 13 & 6 & 19 & 35 & 3 & 38 & 10 \\
\hline Glueck & 1999 & America & 55 & 4 & 59 & 40 & 0 & 40 & 11 \\
\hline Glueck & 2008 & America & 11 & 2 & 13 & 64 & 0 & 64 & 12 \\
\hline Glueck & 2012 & America & 221 & 23 & 244 & 102 & 2 & 104 & 13 \\
\hline Zalavras & 2004 & Greece & 59 & 13 & 72 & 286 & 14 & 300 & 14 \\
\hline
\end{tabular}

Wild, wild homozygote, i.e., the genotype of GG; Leiden, carriers of rare alleles, i.e., the genotype of GA + AA.

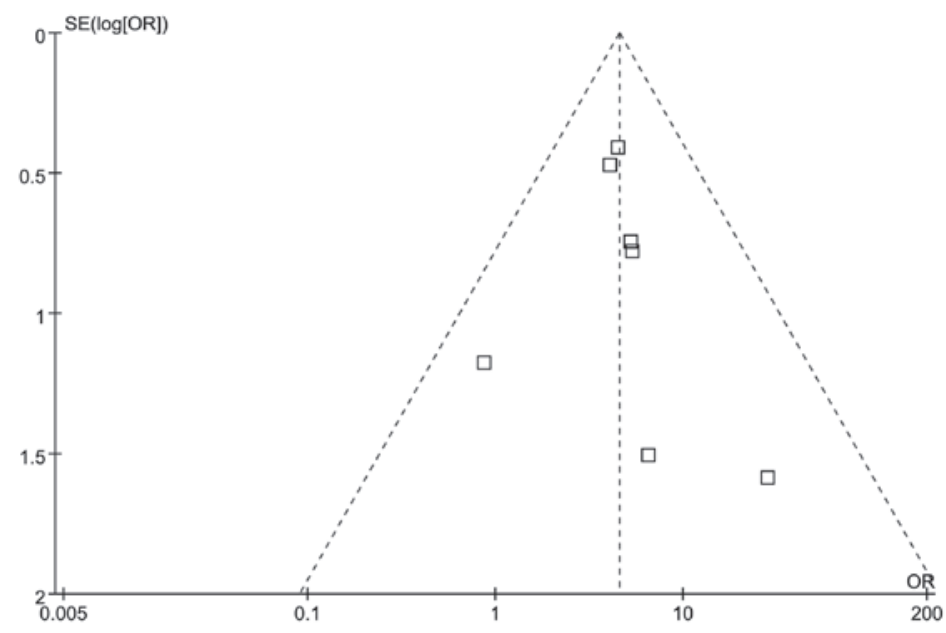

Figure 2. Funnel plot for Factor V Leiden to detect publication bias. Each square represents a separate study for the indicated association.

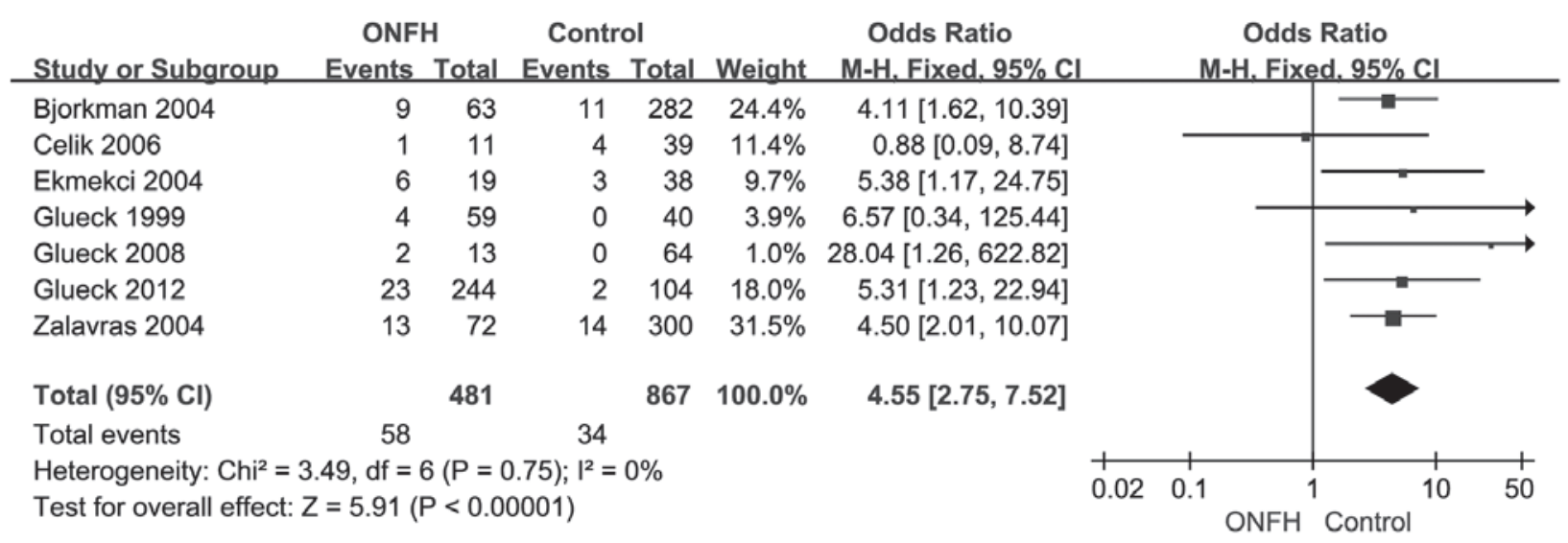

Figure 3. Forest plot for Factor V Leiden mutation of the association between Factor V G1691A polymorphism and osteonecrosis of femoral head (ONFH). Each study is shown by the point estimate of the OR and 95\% CI for the OR.

sion test was used to provide statistical evidence of funnel plot symmetry $(\mathrm{P}=0.65)$, which confirmed no evidence of substantial publication bias (data not shown).

Association of FVL and ONFH. The association between FVL mutation and risk of developing ONFH in the overall population were detected when examining the contrast of FVL vs. wild homozygote $(\mathrm{OR}=4.55,95 \% \mathrm{CI}, 2.75-7.52$, $\mathrm{P}<0.00001)$ and without significant between-study heterogeneity $\left(\chi^{2}=3.49, \mathrm{P}=0.75, \mathrm{I}^{2}=0 \%\right)$. The forest plot of the distribution of the ORs is shown in Fig. 3. A sensitivity analysis was performed by changing the fixed-effects model into the 
random-effects model. The results showed that there was still a significant association when the random-effects model was used $(\mathrm{OR}=4.47,95 \% \mathrm{CI}, 2.71-7.37, \mathrm{P}<0.00001)$ although no results were found for the forest plot of the distribution of the ORs. The results of the fixed- and random-effects models were in agreement with each other.

\section{Discussion}

In recent years, an increased tendency for intravascular coagulation has been proposed as the final pathway in the pathogenesis of osteonecrosis (19) and various environmental and genetic risk factors lead to the interruption of the intraosseous microcirculation supply and osteonecrosis. Intravascular thrombosis is an effect of intravascular coagulation disorder and is considered the cause of non-traumatic osteonecrosis (20). FVL mutation is a most common inherited thrombophilias and causes APC resistance. An association of APC resistance with elevated plasma levels of coagulation markers has been identified, suggesting that this phenotype represented an in vivo hypercoagulable state (21). However, inconsistent results were observed when FVL was investigated in ONFH patients. Considered individually, some studies in our meta-analysis did not reveal a statistically significant association with ONFH (Table I and Fig. 3), possibly due to small sample size and therefore limited statistical power of these individual studies. The largest sample size among the included studies was 372 , while the smallest was only 50 . However, most studies concluded that the FVL polymorphism was a risk factor for ONFH. Therefore, our meta-analysis draws a conclusion that FVL has an effect on ONFH patients.

Based on the results obtained, we established an association between FVL mutation and ONFH, with a cumulative OR of 4.55. Therefore, the carriers of FVL mutation had a 4-fold risk of ONFH when compared with non-carriers. Inclusion and exclusion criteria are a crucial component of a meta-analysis and may affect the results. We were relatively conservative in selecting studies, which likely underestimated the alrealy identified association. Several articles investigating FVL met our criteria, but they were excluded as they could not provide sufficient information for calculating the ORs of the FVL mutation and be extracted (22-24). Consequently, the studies that were included in this meta-analysis strengthened the association between FVL mutation and ONFH.

Of note, the disproportionately large study by Zalavras et al (14) showed a strong association $(\mathrm{OR}=4.5)$ and it contributed $31.5 \%$ of the FVL-positive ONFH cases to our meta-analysis. Nevertheless, the relatively small study by Celik et al (9) showed no association (OR=0.88). One possible reason for the insignificant effect of the present study was the limited sample size. Additionally, the main population included in the meta-analysis was non-Asian. Two studies in which the study subjects included an Asian population and conformed to the included criteria were deleted as they observed no FVL mutation in cases or controls. Chang et al (22) suggested that the FVL mutation is not a genetic risk factor for ONFH in Asians, or at least in the Korean population, which was in agreement with findings by Sun et al (24). The results obtained by these authors were consistent with previous reports, which demonstrated the absence of FVL mutation in the Chinese and Korean populations $(25,26)$. A previous study also showed that Factor V Leiden allele was present in $\sim 5 \%$ of Caucasian individuals (Europeans, Jews, Israeli Arabs and Indians) (27). The reasons for the same polymorphism having different associations influenced by different ethnic populations or studies may be explained by various factors. Firstly, the polymorphism plays a different role in different ethnicities in different genetic backgrounds, as indicated above. There are also a number of other factors that may affect thrombosis, such as the damage of vascular endothelial cells, changes of blood flow and drugs. Additionally, the potential contribution of differences in patient demographic parameters, such as age, gender and disease severity that may lead to varying results should be considered, although this data was not available for the present meta-analysis.

In addition, FVL can be co-inherited with other genetic risk factors to increase the susceptibility to thrombosis in the individual patient population (28). A number of the studies included in this meta-analysis considered multiple genetic risk factors. 5,10-Methylenetetrahydrofolate reductase (MTHFR) is an enzyme that plays a role in the re-methylation of homocysteine and the C667T polymorphism is involved in the elevated plasma total homocysteine level (29). Furthermore, it has been proven that coinheritance of MTHFR and FVL was likely to increase the risk of venous thromboembolism (30). Another such polymorphism is the prothrombin gene G20210A mutation, which is associated with a $20-50 \%$ increase in prothrombin plasma levels (31). In addition to the FVL polymorphism, two studies $(9,11)$ investigated the MTHFR C667T polymorphism and five studies $(8-10,13,14)$ investigated the prothrombin gene G20210A mutation association with ONFH. However, results of most studies indicated a negative conclusion, which reported that MTHFR C667T polymorphism and prothrombin gene G20210A mutation were not risk factors in ONFH. However, a limited sample size may have been used and therefore a lower power to detect the true association. Therefore, larger studies are necessary to determine whether these polymorphisms and mutations serve as risk factors for ONFH.

Nevertheless, there were limitations to this meta-analysis. Firstly, the sample size of studies included was relatively small and more studies containing a large sample size are required. Secondly, most control groups were not uniformly defined. Case and control groups were not age- and gender-matched, which might increase the selection bias. Thirdly, we focused mainly on English and Chinese studies, thus, language bias may also be a relevant factor. Fourthly, the meta-analysis remains a retrospective investigation that is subject to the methodological deficiencies of the included studies. Additionally, no publication bias was detected, indicating that the total pooled OR might be unbiased.

In conclusion, the present meta-analysis has demonstrated the association between FVL polymorphism and ONFH susceptibility. However, to reach a definitive conclusion, large sample studies including different ethnic groups with age- and gender-matched groups and multiple gene polymorphism detection should be considered. Additional investigations focusing on the gene-gene and gene-environment interaction in the process of pathogenesis should also be conducted. 


\section{References}

1. Malizos KN, Karantanas AH, Varitimidis SE, Dailiana ZH, Bargiotas K and Maris T: Osteonecrosis of the femoral head: etiology, imaging and treatment. Eur J Radiol 63: 16-28, 2007.

2. Jones LC and Hungerford DS: Osteonecrosis: etiology, diagnosis, and treatment. Curr Opin Rheumatol 16: 443-449, 2004.

3. Lafforgue P: Pathophysiology and natural history of avascular necrosis of bone. Joint Bone Spine 73: 500-507, 2006.

4. Rosing J and Tans G: Coagulation factor V: an old star shines again. Thromb Haemost 78: 427-433, 1997.

5. Bertina RM, Koeleman BPC, Koster T, et al: Mutation in blood coagulation factor $\mathrm{V}$ associated with resistance to activated protein C. Nature 369: 64-67, 1994.

6. Dentali F, Ageno W, Bozzato S, et al: Role of factor V Leiden or G20210A prothrombin mutation in patients with symptomatic pulmonary embolism and deep vein thrombosis: a meta-analysis of the literature. J Thromb Haemost 10: 732-737, 2012.

7. Dowaidar M and Settin A: Risk of myocardial infarction related to factor V Leiden mutation: a meta-analysis. Genet Test Mol Biomarkers 14: 493-498, 2010.

8. Bjorkman A, Svensson PJ, Hillarp A, Burtscher IM, Runow A and Benoni G: Factor V leiden and prothrombin gene mutation: risk factors for osteonecrosis of the femoral head in adults. Clin Orthop Relat Res 452: 168-172, 2004.

9. Celik A, Tekis D, Saglam F, et al: Association of corticosteroids and factor V, prothrombin, and MTHFR gene mutations with avascular osteonecrosis in renal allograft recipients. Transplant Proc 38: 512-516, 2006

10. Ekmekci Y,Keven K, Akar N, et al: Thrombophilia and avascular necrosis of femoral head in kidney allograft recipients. Nephrol Dial Transplant 21: 3555-3558, 2006.

11. Glueck CJ, Fontaine RN, Gruppo R, et al: The plasminogen activator inhibitor-1 gene, hypofibrinolysis, and osteonecrosis Clin Orthop Relat Res 366: 133-146, 1999.

12. Glueck CJ, Freiberg RA, Boppana S and Wang P: Thrombophilia, hypofibrinolysis, the eNOS T-786C polymorphism, and multifocal osteonecrosis. J Bone Joint Surg Am 90: 2220-2229, 2008.

13. Glueck CJ, Freiberg RA, Boriel G, et al: The role of the factor V Leiden mutation in osteonecrosis of the hip. Clin Appl Thromb Hemost: June 12, 2012 (Epub ahead of print).

14. Zalavras CG, Vartholomatos G, Dokou E and Malizos KN: Genetic background of osteonecrosis: associated with thrombophilic mutations? Clin Orthop Relat Res 251-255, 2004.

15. Egger M, Davey Smith G, Schneider M and Minder C: Bias in meta-analysis detected by a simple, graphical test. BMJ 315 629-634, 1997.

16. Cochran WG: The combination of estimates from different experiments. Biometrics 10: 101-129, 1954.
17. Higgins JP and Thompson SG: Quantifying heterogeneity in a meta-analysis. Stat Med 21: 1539-1558, 2002 .

18. Higgins JP, Thompson SG, Deeks JJ and Altman DG: Measuring inconsistency in meta-analyses. BMJ 327: 557-560, 2003.

19. Jones JP Jr: Intravascular coagulation and osteonecrosis. Clin Orthop Relat Res 277: 41-53, 1992.

20. Jones JP Jr: Coagulopathies and osteonecrosis. Acta Orthop Belg 65 (Suppl 1): 5-8, 1999.

21. Lowe GD, Rumley A, Woodward M, Reid E and Rumley J: Activated protein C resistance and the FV: R506Q mutation in a random population sample-associations with cardiovascular risk factors and coagulation variables. Thromb Haemost 81: 918-924, 1999.

22. Chang JD, Hur M, Lee SS, Yoo JH and Lee KM: Genetic background of nontraumatic osteonecrosis of the femoral head in the Korean population. Clin Orthop Relat Res 466: 1041-1046, 2008.

23. Kechli AM, Wilimas JA, Pui CH, Park VM, Tonkel S and Deitcher SR: Factor V Leiden and other hypercoagulable state mutations are not associated with osteonecrosis during or after treatment for pediatric malignancy. J Pediatr 134: 310-314, 1999.

24. Sun W, Li ZR, Shi ZC, et al: Hematological changes and related gene mutation of post-severe acute respiratory syndrome patients with osteonecrosis. Zhonghua Yi Xue Za Zhi 86: 442-445, 2006 (In Chinese)

25. Jun ZJ, Ping T, Lei Y, Li L, Ming SY and Jing W: Prevalence of factor V Leiden and prothrombin G20210A mutations in Chinese patients with deep venous thrombosis and pulmonary embolism. Clin Lab Haematol 28: 111-116, 2006.

26. Kim YW, Yoon KY, Park S, Shim YS, Cho HI and Park SS: Absence of factor V Leiden mutation in Koreans. Thromb Res 86: 181-182, 1997.

27. De Stefano V, Chiusolo P, Paciaroni K and Leone G: Epidemiology of factor V Leiden: clinical implications. Semin Thromb Hemost 24: 367-379, 1998.

28. Rosendorff A and Dorfman DM: Activated protein C resistance and factor V Leiden: a review. Arch Pathol Lab Med 131: 866-871, 2007.

29. Frosst P, Blom HJ, Milos R, et al: A candidate genetic risk factor for vascular disease: a common mutation in methylenetetrahydrofolate reductase. Nat Genet 10: 111-113, 1995.

30. Eldibany MM and Caprini JA: Hyperhomocysteinemia and thrombosis: an overview. Arch Pathol Lab Med 131: 872-884, 2007.

31. Rosendaal FR, Doggen CJ, Zivelin A, et al: Geographic distribution of the $20210 \mathrm{G}$ to A prothrombin variant. Thromb Haemost 79: 706-708, 1998. 